\title{
Le système éducatif tunisien
}

\section{Cécile de Bouttemont}

\section{(2) OpenEdition}

Journals

Édition électronique

URL : https://journals.openedition.org/ries/1928

DOI : $10.4000 /$ ries. 1928

ISSN : 2261-4265

\section{Éditeur}

France Education international

\section{Édition imprimée}

Date de publication : 1 avril 2002

Pagination : 129-136

ISBN : 2-84520-553-8

ISSN : $1254-4590$

\section{Référence électronique}

Cécile de Bouttemont, « Le système éducatif tunisien », Revue internationale d'éducation de Sèvres [En ligne], 29 | avril 2002, mis en ligne le 25 novembre 2011, consulté le 05 juillet 2021. URL : http:// journals.openedition.org/ries/1928; DOI : https://doi.org/10.4000/ries.1928

Ce document a été généré automatiquement le 5 juillet 2021

(c) Tous droits réservés 


\title{
Le système éducatif tunisien
}

\author{
Cécile de Bouttemont
}

\section{La République tunisienne}

\author{
Superficie : $164000 \mathrm{~km}^{2}$ \\ Population : 9,2 millions d'habitants \\ Croissance annuelle : $1,1 \%$ \\ Densité : $57,8 \mathrm{hab} / \mathrm{km}^{2}$ \\ Capitale : Tunis \\ Économie : \\ PIB total (2000) : $5 \%$ \\ PIB par habitant : $5,957 \$$ \\ Religion : musulmans sunnites à $99 \%$ \\ Langues : arabe dialectal, arabe littéraire, français
}

1 La Tunisie est l'une des plus anciennes entités politiques du Maghreb. Ouvert sur le monde méditerranéen, le pays a accueilli sur son sol de nombreuses civilisations: phénicienne, romaine et arabo-musulmane. Premier pays du Maghreb a avoir été arabisé, la Tunisie est devenue indépendante en 1956 après 73 ans de protectorat français.

2 Elle apparaît comme un pôle de stabilité dans la région et bénéficie d'une position particulière au sein de l'aire arabo-musulmane du fait de ses spécificités à la fois culturelles et économiques. Ainsi, sa population se caractérise par une grande homogénéité religieuse et linguistique. Musulmane, elle ignore les clivages religieux qui affecte le Machrek. Arabe elle ne connaît pratiquement pas les clivages linguistiques (arabophones/berbérophones) observables en Algérie et au Maroc.

3 Comparée à ses voisins marocain, algérien ou libyen, pourtant dotés d'une manne pétrolière qui lui fait défaut, la Tunisie affiche une réussite économique appréciable. La croissance économique a atteint $5 \%$ en moyenne ces dernières années et le revenu 
annuel par habitant s'élève à $13200 \mathrm{FF}$, ce qui la place en tête des pays du Maghreb et au $8^{\mathrm{e}}$ rang en Afrique ${ }^{1}$.

4 En matière éducative, la Tunisie a également enregistré des améliorations notables ces dernières années et fait figure de bon élève du Maghreb. Son système éducatif reste néanmoins confronté à de nombreux défis² .

\section{Depuis l'indépendance, un bilan globalement positif}

5 Depuis l'indépendance, le système éducatif tunisien s'est nettement amélioré et affiche aujourd'hui des résultats positifs en regard des autres pays du Maghreb observables en fonction d'indications statistiques.

6 Le taux de scolarisation a fortement augmenté. Ainsi, $67 \%$ de la classe d'âge 13-19 ans était scolarisée en 2000-2001 contre $54 \%$ en 1995-96. Par ailleurs, le nombre d'écoles du $1^{\text {er }}$ cycle de l'enseignement de base est passé de 2319 à 4465 entre 1975-2000. Dans la même période, le nombre des établissements correspondants au $2^{\mathrm{e}}$ cycle ajoutés à ceux de l'enseignement secondaire passe de 171 à $1019^{3}$. L'ouverture de 14 nouveaux établissements universitaires était programmée pour la rentrée $2000^{4}$.

7 Le taux d'analphabétisme a considérablement diminué, passant de $84 \%$ en 1956 à $35 \%$ en 1990. Ce taux, évalué à $50 \%$ au Maroc et à $42 \%$ en Algérie, est le plus bas du Maghreb.

8 Le niveau général d'instruction de la population active a augmenté, conséquence d'une plus grande efficacité du système éducatif. Moins de $1 \%$ des élèves entrant dans le primaire en 1972-1973 ont obtenu le baccalauréat, alors que $44 \%$ de ceux entrant en 2000-2001 devraient l'obtenir' ${ }^{6}$. En 2001, le nombre de candidats au baccalauréat était de $6 \%$ supérieur à celui de l'an 2000, et parmi eux, les filles représentaient $53 \%$. Dans l'enseignement supérieur, quelque 25000 bacheliers en moyenne sont admis chaque année à l'université, où le nombre d'inscrits est passé de 30000 en 1980 à plus de 200000 en 2000, l'objectif étant d'atteindre 300000 en $2010^{7}$.

9 L'éducation en Tunisie a toujours été considérée comme un facteur de développement économique majeur. Même en période de difficultés économiques, la Tunisie a consacré environ $7 \%$ du PIB par an à l'éducation, soit près de $25 \%$ du budget général, ce qui la place en tête des pays du Maghreb

10 Tous ces résultats sont le fruit d'une politique volontariste d'adaptation du système éducatif tunisien depuis son indépendance en 1956.

11 Dès l'obtention de l'indépendance, Habib Bourguiba, premier président de la République Tunisienne, engage une nouvelle ère en matière d'éducation. S'appuyant sur les structures mises en place sous le protectorat, il mène une politique de scolarisation de masse en rendant la scolarité obligatoire.

12 À la fin des années 80, le bilan éducatif de Bourguiba demeure contrasté. Le taux d'analphabétisme a sensiblement diminué (en 1984, près de $50 \%$ de la population âgée de 10 ans est réputée savoir lire et écrire, contre seulement $15 \%$ en 1956). Il reste cependant très élevé en milieu rural et globalement massif chez les femmes. En 1975, seule la moitié d'entre elles était scolarisée9.

13 L'explosion démographique explique pour une bonne part l'augmentation des budgets éducatifs. Quant à l'augmentation des effectifs en primaire, elle ne s'est pas toujours 
accompagnée d'une amélioration qualitative. En effet, le taux d'échec scolaire est élevé : $30 \%$ des filles et $20 \%$ des garçons quittent le système scolaire avant la $6^{\mathrm{e}}$. À certains égards, la généralisation de l'enseignement après l'indépendance a substitué au système fermé à l'entrée, en vigueur à l'époque coloniale, un système de sortie par l'échec : en 1978 sur 100 enfants entrant à l'école primaire, 84 n'achèveront jamais le $1^{\text {er }}$ cycle et un seul parviendra au baccalauréat. Quant au supérieur, seuls $8 \%$ de la tranche d'âge des 18-24 ans ont la chance d'y accéder ${ }^{10}$.

14 Enfin, sur les 350000 chômeurs (16\%) de la population active, 70 \% sont des jeunes. L'impact de l'éducation sur l'obtention d'un emploi est problématique et le niveau d'instruction des chômeurs s'élève au rythme de celui de l'ensemble de la population active $^{11}$.

En 1987, le président Bourguiba est destitué par un coup d'État et remplacé par le général Ben Ali. L'avènement de cette $\mathrm{II}^{\mathrm{e}}$ république tunisienne ouvre une phase de croissance économique qui débute avec le plan d'ajustement structurel du FMI appliqué la même année.

16 À partir de 1989, le ministre de l'Éducation, Mohammed Charfi, lance une vaste réforme pour rénover le système éducatif. Ses objectifs sont les suivants : améliorer la qualité de l'enseignement, rechercher la meilleure adéquation possible entre la formation et l'emploi, ouvrir l'école sur la société et le monde moderne. Une loi promulguée en 1991 définit les principales mesures :

- le système scolaire est désormais obligatoire durant neuf années au lieu de six. La réforme instaure un enseignement de base comportant un premier cycle de six ans et un second cycle de trois ans, et un enseignement secondaire de quatre ans. L'affichage d'un «enseignement de base " marque clairement l'ambition d'assurer un socle de formation jusqu'à 16 ans à l'ensemble d'une génération ;

- il intègre l'enseignement technique dès le collège et propose un bac technique de qualité ;

- il impose l'apprentissage du français dès la $3^{\mathrm{e}}$ année du primaire (cette mesure vise à réhabiliter l'enseignement de cette langue qui demeure indispensable pour réussir ses études et trouver des débouchés professionnels). Dès le premier et le second cycle de l'enseignement de base, toutes les matières sont enseignées en arabe. Le français, dès la $3^{\mathrm{e}}$ année du $1^{\mathrm{er}}$ cycle et l'anglais à partir de la $8^{\mathrm{e}}$ année, sont enseignées en tant que disciplines ;

- la formation des enseignants est repensée. Une nouvelle école normale, calquée sur le modèle français, forme pendant deux ans les instituteurs, tandis que les professeurs du secondaire ne peuvent désormais exercer qu'à la condition d'avoir obtenu un diplôme sanctionnant deux années d'études supérieures.

\section{Un système éducatif marqué par le bilinguisme}

17 Comme dans les autres pays du Maghreb, le système éducatif doit composer avec une situation linguistique complexe qui conditionne fortement son organisation et son évolution.

18 Ainsi, la langue maternelle des enfants tunisiens est l'arabe dialectal, une langue orale, qui n'est ni uniforme ni homogène. La langue sur laquelle se fonde l'identité tunisienne et son appartenance à la culture arabo-musulmane est l'arabe classique, langue d'enseignement officielle. Cette langue s'avère aujourd'hui encore mal adaptée à 
l'enseignement des matières scientifiques et techniques pour plusieurs raisons: problème de formation des maîtres, absence de programmes et de matériels, difficulté à transcrire les concepts scientifiques français dans un arabe harmonisé. Enfin, les autorités et les médias utilisent l'arabe moderne international qui, malgré un lexique commun, est encore différent des deux autres.

Dans ce contexte, le français bénéficie d'une position particulière et ambiguë. En effet, il continue d'être ressenti comme la langue du colonisateur, véhicule d'un mode de vie et de valeurs importés. Mais il est essentiel comme moyen d'ouverture. Il demeure indispensable pour accéder à l'enseignement scientifique et à certaines professions, et plus généralement pour participer au développement de l'économie tunisienne de plus en plus ouverte sur l'international. D'autre part, le français ne peut aujourd'hui être complètement exclu du patrimoine national en raison d'une abondante littérature tunisienne d'expression française et de l'utilisation de cette langue dans les milieux intellectuel et médiatique.

Pour ajouter à cette complexité, l'exposition des écoliers tunisiens au français varie considérablement selon qu'ils vivent soit dans des grandes villes côtières ou touristiques, soit dans les zones rurales où le français s'apparente plutôt à une langue étrangère. D'autre part, elle dépend des origines sociales des enfants: les milieux intellectuels et aisés sont plus fréquemment bilingues que les familles populaires.

Partagés entre la volonté d'affirmer l'identité tunisienne à travers la langue arabe et le constat que l'étude du français s'avère indispensable, les gouvernements tunisiens successifs mènent des politiques linguistiques variables qui, compte tenu de l'importance $\mathrm{du}$ français dans l'enseignement supérieur, ont profondément conditionné l'évolution du système éducatif tunisien.

22 Ainsi, au lendemain de l'indépendance, en 1958, le plan Messaadi, reprend, en l'adaptant et le généralisant, le modèle institué sous le protectorat, destiné à former un individu parfaitement bilingue: le français, introduit dès la deuxième année de scolarité croît rapidement jusqu'à devenir prépondérant à la fin du cycle primaire. Ce système s'adresse initialement à une élite et ne peut donc sans difficultés être dispensé tel quel à l'ensemble de la population tunisienne.

23 À partir de 1970, est adoptée une réforme visant à l'arabisation de l'enseignement. La primauté est alors donnée à la langue arabe qui devient la langue véhiculaire de toutes les disciplines. Le français devient langue d'appoint, langue d'information et de communication permettant l'accès à la culture scientifique et technique. En parallèle, l'arabisation de l'enseignement technique et scientifique est lancée et constitue un axe important de la politique éducative. Elle se heurte néanmoins à de sérieuses difficultés.

24 Avec cette réforme, le modèle didactique évolue. On passe ainsi d'un modèle qui souligne la dimension culturelle et fait jouer un rôle essentiel à l'étude de la littérature à des conceptions marquées par l'enseignement des langues étrangères qui mettent en avant des objectifs d'ordre « communicationnels».

La réforme de 1991 réhabilite la place du français en rendant l'enseignement du français obligatoire dès la $3^{\mathrm{e}}$ année du primaire et en imposant des horaires d'enseignement beaucoup plus lourds que ceux d'une simple langue étrangère. Le français est ainsi enseigné de $9 \mathrm{~h}$ à $11 \mathrm{~h}$ par semaine entre la $3^{\mathrm{e}}$ et la $6^{\mathrm{e}}$ année du $1^{\mathrm{er}}$ cycle de l'école de base et $5 \mathrm{~h}$ les trois dernières années du $2^{\text {nd }}$ cycle de l'école de base. L'anglais n'apparaît qu'à la fin du second cycle avec $2 \mathrm{~h}$ par semaine. Sur le plan 
didactique s'affirme la volonté de trouver un équilibre pour associer la dimension culturelle et les apprentissages opérationnels ${ }^{12}$.

Ces allers-retours entre des conceptions différentes du statut du français se traduisent dans les faits par la persistance de certaines contradictions dans l'enseignement de cette langue, qui rend son enseignement, par certains aspects, inadapté.

D'une part les programmes d'enseignement du français s'avèrent relativement ambitieux et sont souvent inapplicables dans la réalité scolaire car leurs exigences sont souvent calquées sur celles des programmes français de langue maternelle. À titre d'exemple, certaines propositions de lecture longue en $4^{\mathrm{e}}$ année du secondaire (Eugénie Grandet, Vol de nuit...) sont des œuvres au programme de la classe de $3^{\mathrm{e}}$ en France. D'autre part, alors que l'enseignement tente de développer une compétence principalement communicative, les élèves continuent d'être évalués sur leurs travaux écrits.

Par ailleurs, l'uniformité du matériel employé ne tient pas assez compte de la situation sociale des écoles, alors même que l'exposition des écoliers tunisiens au français varie considérablement selon les régions et les milieux.

Enfin, les horaires accordés au français restent significatifs d'un manque de continuité dans le projet éducatif et sont sources de " points de rupture » dans le cursus scolaire ${ }^{13}$. Ainsi, le passage du $1^{\text {er }}$ degré de l'enseignement de base au second degré constitue un seuil difficile du fait de la réduction du nombre d'heures consacrées à l'enseignement $\mathrm{du}$ français $(11 \mathrm{~h}$ à $5 \mathrm{~h})$ alors que les élèves sont confrontés à la compréhension de textes plus difficiles ${ }^{14}$. Le passage de l'école de base à l'enseignement secondaire pose un autre problème : objet d'enseignement et bénéficiant d'horaires très importants, le français devient véhicule d'enseignement dans le secondaire (matières scientifiques et techniques). Enfin au niveau universitaire, la section lettres, qui met l'accent sur la littérature, est susceptible d'accueillir les élèves du secondaire originaires de cette section. Or, dès le secondaire, les horaires sont progressivement réduits contrairement à ceux de la section scientifique.

30 La complexité de la situation linguistique en Tunisie, les changements d'orientation successifs en matière de politique linguistique et les inadaptations qui subsistent dans l'enseignement du français ont eu plusieurs conséquences :

- partagés durant toute leur scolarité entre deux langues au statut variable et indéterminé, les jeunes Tunisiens sont bien souvent dépourvus d'une réelle maîtrise de l'une ou l'autre langue ;

- concernant le français, si le nombre des locuteurs a significativement augmenté avec l'essor démographique et la généralisation de l'enseignement, on constate toutefois une baisse de la part des francophones d'éducation et de culture. L'évolution du modèle didactique vers le développement de compétences « communicationnelles » a entraîné une moindre maîtrise de la langue ;

- enfin, compte tenu de la place que le français occupe encore dans l'enseignement supérieur et des contradictions de son enseignement dans le secondaire, il constitue, de fait, un frein à l'accès de certains élèves aux matières scientifiques et techniques.

31 Au sein d'une identité tunisienne qui se cherche, deux lignes de fracture se profilent. La situation de la langue arabe engendre une profonde dichotomie entre langue parlée et écrite ce qui accentue la scission entre l'État et la société. La langue arabe administrative se présente sans références communautaires car elle n'est parlée par personne en dehors de l'usage officiel. «Cette diglossie qui préexiste à la colonisation a 
été accentuée par le nationalisme (...) faisant de l'utilisation de l'arabe moderne l'instrument symbolique de l'affirmation d'une identité arabe $»^{15}$. Par ailleurs, le bilinguisme entraîne aussi des situations paradoxales : tout en se servant de la langue arabe moderne pour consolider une légitimité politique vacillante, l'État continue d'utiliser le français comme instrument privilégié d'accès au pouvoir. La principale conséquence de ces contradictions est la constitution d'une identité culturelle pas toujours homogène, divisée selon des lignes de fracture «qui opposent l'oral à l'écrit, la masse populaire à l'État, l'individu à la société civile ${ }^{16}$.

\section{Les défis du développement et de l'ouverture économique}

Depuis les années 90, l'économie tunisienne s'est engagée dans une phase de préparation de son intégration à l'économie mondiale. Membre de l'Organisation Mondiale du Commerce, la Tunisie a également signé un accord d'association avec l'Union Européenne en juillet 1995 qui prévoit la mise en place progressive d'une zone de libre échange d'ici à 2008.

Pour renforcer sa compétitivité dans ce contexte et accélérer son développement économique et social, la Tunisie s'est engagée dans une réforme de société globale dont le système éducatif constitue un maillon clé. Il reste confronté à d'importants défis : poursuivre l'effort d'éducation de base - lutte contre l'abandon scolaire, lutte contre l'analphabétisme dans les campagnes, promotion de l'éducation de la femme; améliorer le niveau de qualification des jeunes diplômés disponibles sur le marché du travail et développer les filières dont le pays a besoin (TIC notamment); adapter les formations aux besoins des entreprises pour réduire le chômage en fournissant un emploi à tous les jeunes qui entrent chaque année sur le marché du travail ${ }^{17}$.

Pour accélérer la mutation du système éducatif, le gouvernement a engagé plusieurs réformes internes et a choisi de s'appuyer très largement sur la coopération internationale.

\section{Réformes internes}

En matière d'enseignement de base, le gouvernement a prévu de développer l'éducation préscolaire en zone rurale et populaire. Par ailleurs, il souhaite accentuer la décentralisation. En effet, le pouvoir local reste très limité dans un pays historiquement centralisé et peu étendu géographiquement. Le pouvoir est détenu par des gouverneurs nommés par le Président de la République à la tête de 23 gouvernorats.

En ce qui concerne l'enseignement supérieur, le budget est en nette augmentation ces dernières années. En 2000, il a ainsi augmenté de 13,6 \% par rapport à 1999 ${ }^{18}$. L'objectif est de recruter 1000 enseignants par an, alors qu'on en compte actuellement 10000 . Un effort tout particulier sera fait sur les filières d'ingénieur en vue de fournir à l'économie nationale des compétences hautement qualifiées.

D'autre part, pour favoriser l'adaptation des études universitaires au marché de l'emploi, le gouvernement souhaite promouvoir la collaboration université-entreprise. Les instituts supérieurs d'études technologiques (équivalents des IUT français) constituent un modèle de cette politique de partenariat initiée par le ministère de 
l'Enseignement supérieur depuis 1995. Pour les autres établissements d'enseignement et de recherche, il s'agit de constituer des cellules de liaison pour assurer la communication avec les entreprises.

Enfin, pour préparer la Tunisie à la concurrence des produits agricoles étrangers (ouverture prévue pour 2008), une réforme est envisagée pour former une maind'œuvre spécialisée en fonction des besoins réels de la région d'implantation de l'établissement agricole permettant une qualification régionale.

\section{Favoriser la coopération en éducation}

\section{L'Union du Maghreb Arabe (UMA)}

En dépit de relations diplomatiques fragiles avec ses voisins, la Tunisie a engagé un processus de coopération en éducation. Lors d'une déclaration commune à Nouakchott, en février 2001, les ministres de l'éducation des pays membres de l'UMA ${ }^{19}$ ont recommandé "la redynamisation des structures dans le domaine des ressources humaines ». Huit conventions et accords ont été signés par les chefs d'État entre 1991 et 1994 ainsi que la création de quatre institutions communautaires dont une universitée ${ }^{20}$. Enfin, lors de la clôture de la Commission tuniso-libyenne de l'Éducation, une série de recommandations ont été prises visant à enrichir la coopération entre Tunis et Tripoli. Cette coopération concerne entre autres le jumelage des institutions éducatives et l'échange de professeurs ${ }^{21}$.

\section{La France}

41 En plus de liens historiques avec la Tunisie, la France est le premier partenaire commercial de la Tunisie en fournissant plus d'un quart des importations et recevant environ $30 \%$ des exportations. Parmi les initiatives de coopération en éducation on peut noter les nombreux partenariats et échanges pédagogiques entre les deux pays.

\section{L'Union européenne}

Elle reste le principal partenaire commercial. L'Unioneuropéenne représente plus des trois quarts des débouchés et des sources d'approvisionnement de la Tunisie, alors que celle-ci représente moins de $1 \%$ des exportations extracommunautaires.

Sur le plan éducatif, le président de la Commission Européenne, Romano Prodi, et le ministre tunisien de la Coopération Internationale et de l'Investissement extérieur, Fehti Merdassi, ont signé le 11 janvier à Tunis un projet pour la période 2000-2002, bénéficiant de 40 millions d'euros pour promouvoir l'éducation de base. Afin de développer l'efficacité du système éducatif, les principaux objectifs fixés s'articulent autour des axes suivants :

- améliorer l'efficacité du système éducatif en agissant sur l'échec scolaire ;

- généraliser les prestations éducatives au milieu rural ;

- modifier les pratiques pédagogiques en instaurant une culture de l'évaluation;

- renforcer une réelle décentralisation/déconcentration pour encourager l'initiative et l'innovation au niveau des régions et des établissements scolaires ;

- généraliser le recours aux supports pédagogiques modernes et aux technologies informatiques. 


\section{NOTES}

1. Les Échos : «Faux-semblants tunisiens », 27 mars 2000, p. 63.

2. Cet article s'appuie sur les analyses et les conclusions présentées dans L'enseignement du français en Tunisie, rapport présenté par Alain Boissinot, Simone Delesalle, Pierre Frantz, Yves Guérin, Bruno Halff, Monique Maquaire et réalisé à la demande des autorités tunisiennes dans le cadre de la coopération franco-tunisienne. Centre international d'études pédagogiques, Sèvres, 15 juin 2001.

3. L'enseignement du français en Tunisie, 15 juin 2001, Centre international d'études pédagogiques, p. 5.

4. Dépêche de l'AFP, 9 juillet 2001.

5. En direct de Tunis ", JDI, février 2001, n 6, p. 74.

6. Rapport Boissinot, p. 5.

7. Dépêche AFP, 9 juillet 2001.

8. Annuaire statistique de l'UNESCO, 1999.

9. Encyclopédie Encarta 99, CD-Rom.

10. id.

11. Les Échos : «Faux-semblants tunisiens », 27 mars 2000, p. 63.

12. Rapport Boissinot.

13. id. p. 11.

14. id.

15. Soraya Tlatli, «L'ambivalence linguistique dans la littérature maghrébine d'expression française » in French Review, vol 72, n 2, p. 300.

16. id.

17. L'économie marocaine crée 125000 emplois par an alors que 180000 diplômés se présentent sur le marché du travail.

18. AEF, 21/02/2001.

19. Étaient présents les ministres de l'Éducation algérien, marocain, tunisien, et mauritanien.

20. AFP, 15 février 2001.

21. AFP, 25 avril 2001.

INDEX

Mots-clés : système éducatif

Index géographique : Tunisie

\section{AUTEUR}

\section{CÉCILE DE BOUTTEMONT}

Documentaliste au Centre de ressources documentaires du CIEP 